\section{PANCREAS}

\section{Islets form as peninsulas}

Pancreatic islets were previously thought to assemble away from pancreatic ducts via a dispersalaggregation model. Research published in Cell reveals a new model of islet morphogenesis in which $\alpha$-cells and $\beta$-cells form layered peninsular structures.

"We performed this study to get a detailed understanding of how pancreatic islets form," explains corresponding author Douglas Melton. "This information could help us in our quest to generate pancreatic islets from human stem cells."

The researchers mapped early pancreatic islet formation in pancreata extracted from neurogenin 3 (a marker of endocrine differentiation; NGN3)-eGFP reporter mice embryos using single cell RNA sequencing. Importantly, $\alpha$-cells appeared earlier than $\beta$-cells in this model, which is consistent with previous reports.

Novel methods of statistical analyses developed by the team were used to track the developmental fate of $\mathrm{NGN}^{+}$cells and to model temporal gene expression programmes. Notably, undifferentiated, early cells have an endocrine-progenitor gene expression programme and the onset of cell specification is marked by a switch to a pan-endocrine expression programme. Following this, differentiating islet cells show a gradual accumulation of cell-specific gene expression programmes

Using immunostaining and tissue clearing, the researchers visualized embryonic pancreata in $3 \mathrm{D}$. Strikingly, endocrine progenitors were seen to form budding peninsula shapes, which grow over time, with older cells being pushed outwards by newer cells. The peninsulas form a layered structure that faces away from epithelial cords, with $\alpha$-cells lying on top of $\beta$-cells.

Finally, the researchers generated similar human endocrine peninsulalike structures in vitro by directing the differentiation programmes of human embryonic stem cells.

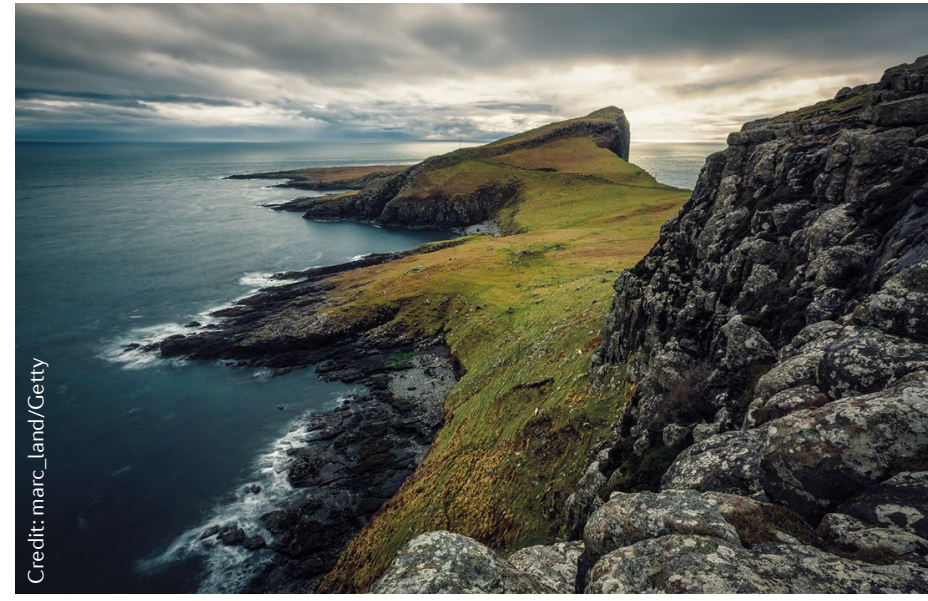

"These findings provide hints about how to improve the production of islet cells from human stem cells," concludes Melton. "The results indicate the order in which cells appear from progenitors, the temporal expression of some key genes and the physical arrangements of cells within a mature islet."

Shimona Starling

form budding

peninsula

shapes

ORIGINAL ARTICLE Sharon, N. et al. A peninsular structure coordinates asynchronous differentiation with morphogenesis to generate pancreatic islets. Cell https://doi.org/10.1016/j.cell.2018.12.003 (2019)

\title{
Breakfast not beneficial for weight loss
}

Eating breakfast does not increase weight loss, according to a new study published in the BMJ. In addition, when compared with people who did eat breakfast, the authors found that individuals who did not eat breakfast were less likely to over-eat later in the day.

"This study came about because in the clinic I saw a lot of patients with osteoarthritis who were obese, who went on to seek advice on what to do regarding their weight, and almost invariably these patients were told they need to eat breakfast," explains corresponding author Flavia Cicuttini. "Some of these patients complained that they were struggling to eat breakfast because they did not feel hungry - it never made sense to me that we should be asking people to eat when they are not hungry."
Data from observational studies that investigated breakfast eating and obesity suggest that breakfast eaters are less likely to be overweight than non-breakfast eaters and that eating breakfast protects against weight gain. In these studies, however, it was not possible to determine whether breakfast eating or an individual's wider healthy lifestyle resulted in weight loss. "Randomized controlled trials take into account lifestyle factors, which means data from these studies are stronger than data from observational studies," adds Circuttini.

In the present systematic review and meta-analysis of randomized controlled trials, Circuttini and her team found that people who ate breakfast tended to eat $\sim 260$ extra calories per day more than those who did not eat breakfast. In addition, when compared with non-breakfast eaters, breakfast eaters people who ate breakfast tended to eat 260 extra calories per day more than those who did not eat breakfast 5

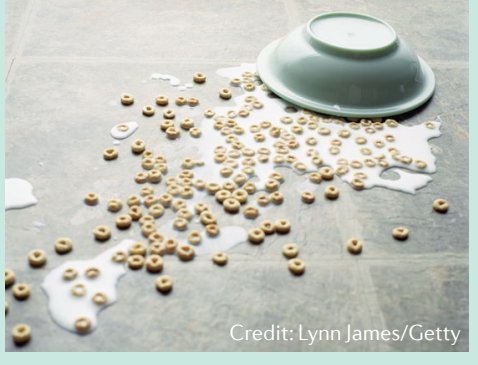

gained an average of $0.44 \mathrm{~kg}$ more body mass during the follow-up period (mean follow-up 7 weeks; range 2-16 weeks). Importantly, there was no evidence to suggest that eating breakfast resulted in improvements to metabolism.

"The key message of the study is that that if a person likes to eat breakfast, that is fine," concludes Circuttini. "However, there is no evidence to suggest that we should be encouraging people to change their eating pattern to include breakfast in order to prevent weight gain."

Alan Morris

ORIGINAL ARTICLE Sievert, K. et al. Effect of breakfast on weight and energy intake: systematic review and meta-analysis of randomised controlled trials. BMJ 364, 142 (2019) 